\title{
BMJ Open Cognitive measures during walking with and without lower-limb prosthesis: protocol for a scoping review
}

\author{
Jing Yuan (D) , ${ }^{1}$ Emily Cline, ${ }^{1}$ Ming Liu, ${ }^{2} \mathrm{He} \mathrm{Huang},{ }^{2}$ Jing Feng ${ }^{1}$
}

To cite: Yuan J, Cline E, Liu M, et al. Cognitive measures during walking with and without lower-limb prosthesis: protocol for a scoping review. BMJ Open 2021;11:e039975. doi:10.1136/ bmjopen-2020-039975

- Prepublication history for this paper is available online. To view these files, please visit the journal online (http://dx.doi. org/10.1136/bmjopen-2020039975).

Received 01 May 2020 Revised 16 December 2020 Accepted 23 January 2021

\section{Check for updates}

\section{(c) Author(s) (or their} employer(s)) 2021. Re-use permitted under CC BY-NC. No commercial re-use. See rights and permissions. Published by BMJ.

${ }^{1}$ Department of Psychology, North Carolina State University, Raleigh, North Carolina, USA

${ }^{2}$ UNC \& NC State Joint Department of Biomedical Engineering, North Carolina State University, Raleigh, North Carolina, USA

Correspondence to

Dr Jing Feng;

jing_feng@ncsu.edu

\section{ABSTRACT}

Introduction Tuning of lower-limb (LL) robotic prosthesis control is necessary to provide personalised assistance to each human wearer during walking. Prostheses wearers' adaptation processes are subjective and the efficiency largely depends on one's mental processes. Therefore, beyond physical motor performance, prosthesis personalisation should consider the wearer's preference and cognitive performance during walking. As a first step, it is necessary to examine the current measures of cognitive performance when a wearer walks with an LL prosthesis, identify the gaps and methodological considerations, and explore additional measures in a walking setting. In this protocol, we outlined a scoping review that will systematically summarise and evaluate the measures of cognitive performance during walking with and without LL prosthesis.

Methods and analysis The review process will be guided and documented by CADIMA, an open-access online data management portal for evidence synthesis. Keyword searches will be conducted in seven databases (Web of Science, MEDLINE, BIOSIS, SciELO Citation Index, ProQuest, CINAHL and PsycINF0) up to 2020 supplemented with grey literature searches. Retrieved records will be screened by at least two independent reviewers on the title-and-abstract level and then the fulltext level. Selected studies will be evaluated for reporting bias. Data on sample characteristics, type of cognitive function, characteristics of cognitive measures, task prioritisation, experimental design and walking setting will be extracted.

Ethics and dissemination This scoping review will evaluate the measures used in previously published studies thus does not require ethical approval. The results will contribute to the advancement of prosthesis tuning processes by reviewing the application status of cognitive measures during walking with and without prosthesis and laying the foundation for developing needed measures for cognitive assessment during walking. The results will be disseminated through conferences and journals.

\section{INTRODUCTION}

Lower-limb (LL) amputation is a major reason for disability in the USA and it is estimated that 1.7 million Americans have experienced amputation, ${ }^{1}$ with the majority of them are on the lower limbs. Traditional passive LL prostheses help amputees to regain the

\section{Strengths and limitations of this study}

- A scoping review is helpful in systematically mapping the current literature from a range of different study designs and types.

- This scoping review will reveal the advantages and disadvantages of the current available measures of cognitive performance during walking and suggest which cognitive processes and measures could be considered during cognitive performance evaluation in prosthesis tuning processes.

- This scoping review is a preliminary step to examine the methods of assessing cognitive performance during walking, it does not examine findings on how the results of cognitive performance evaluation can be incorporated into automatic prosthesis tuning system.

- Compared with systematic reviews or meta-analysis as suggested by Preferred Reporting Items for Systematic Review and Meta-Analysis Protocols, a less formal quality assessment of included articles will be performed given the planned effort being a scoping view of cognitive measures used in a broad range of studies rather than evaluating the findings of these studies.

capability to walk upright, but cannot provide sufficient active torque, which is needed to generate the effective gait as able-bodied. ${ }^{2}$ In the last decade, powered LL prostheses have become commercially available and can mimic dynamical properties of biology joints in a more accurate way. ${ }^{3}$ To ensure good gait performance, the dynamical properties of these advanced devices have to be 'tuned' specifically for each individual participant. ${ }^{4}$ The tuning process is currently carried out manually and heuristically by a tuning expert, a prosthetist from the manufacturers with special training, based on gait performance and user feedback. Because the prosthetists have to tune multiple control parameters, the manual tuning procedure is often tedious and time-consuming. The sparsity of qualified tuning experts also drives up the cost of the related procedure. 
Recently, researchers have developed various approaches to automate the tuning procedure, including expert systems, ${ }^{5}$ reinforcement learning ${ }^{6}$ and humanin-the-loop optimisation. ${ }^{7}$ Even though the existing approaches to the amputee-prosthesis systems manage to ensure the desired physical performance (eg, desired joint kinematics or reduced metabolic cost) in walking, the efficiency of the tuning process is still low and the subjective feedback from the amputee users is usually ignored. ${ }^{8}$

Human's adaptation processes during wearing prostheses are subjective and the efficiency largely depends on one's internal mental processes (eg, preference, perception and cognition). ${ }^{9-11}$ Therefore, beyond physical motor performance, prosthesis personalisation should also consider the wearer's preference and cognitive performance during walking to examine the effectiveness and safety of prosthesis use by individual wearers in the walking processes, which in turn could potentially inform the prosthesis tuning processes in the long run. To achieve this, knowing how to properly quantify the cognitive performance during walking is necessary. However, in the current literature, it is not clear which and how the cognitive performance in a prosthesis wearer could be measured and quantified while the wearer is walking. One structured review was conducted on the dual-task paradigm during standing or walking in the LL amputee population in the literature up to May 2017. ${ }^{12}$ However, dual-task paradigm is not the only way to measure cognition during walking and usually dual-tasks are used as a loading task to test gait performance but not to quantify cognition. Other behavioural measures such as eye-tracking ${ }^{13}$ or psychophysiological measures such as electroencephalogram ${ }^{14}$ or heart rate $^{15}$ are emerging potential quantifiable measures of cognitive processes during walking. Therefore, the current project aims to cover a wider range of cognitive measures beyond the dual-task paradigm and incorporates publications published after 2017.

In addition, to our knowledge, the types of cognitive processes investigated during walking in people with LL prosthesis have been limited. For example, the dual-task method is usually adopted to test the effect of cognitive load on gait performance. Understanding of various cognitive aspects (eg, attention, perception) is necessary to depict a comprehensive picture of performance in daily tasks and scenarios (eg, detecting obstacles and hazards when walking on a road, walking and talking, walking with limited visibility due to weather conditions). Reviewing additional cognitive measures used in the field without using prosthesis can provide further information on the future directions of the choices of cognitive measures, cognitive performance evaluation or cognitive measures development in the prosthesis area. Therefore, we also plan to extend the scope and review the cognitive measures during walking without prosthesis to see if there are quantifiable measures that can be potentially adopted into the prosthesis field.

\section{Objectives}

In sum, the objectives of this project are to review: (1a) what cognitive processes have been investigated in the process of walking with LL prosthesis; (1b) how those cognitive processes are measured and what are their advantages and disadvantages in terms of quantification; (2a) which additional cognitive processes are investigated during walking in general (ie, without prosthesis); and (2b) which additional cognitive measures are used during walking in general and whether they have the potential to be adopted into prosthesis field.

\section{METHOD}

All the data and scoping review processes are guided, managed and recorded through CADIMA, ${ }^{16}$ an openaccess online data management portal for evidence synthesis. As guided by CADIMA, this method section contains six sections: search strategy (the keywords and databases that will be searched), scoping exercise (a demonstration of the effectiveness of our search strategy), study inclusion criteria (what criteria we will hold to screen the papers), study screening (the procedure of abstract and full-text screening), critical appraisal (how we will further assess the study quality), and data extraction (what information we will get from the paper and how we will extract it).

\section{Search strategy}

The following databases will be searched: Web of Science (WOS; including: WOS core databases, MEDLINE, BIOSIS, SciELO Citation Index; from 1900 to 2020), ProQuest Dissertations \& Theses Global (from 1861 to 2020), and EBSCO (including CINAHL and PsycINFO; from 1937 to 2020). Conference papers will be manually searched under specific conference databases such as IEEE Xplore digital library. The first 200 hits in the general full-text search engines such Google Scholar and JSTOR will be checked. In addition to these databases, review papers and meta-analyses identified during the literature screening process will be checked for unidentified but relevant references which will be documented and added into screening processes. In cases where a known lab is conducting relevant research but data have not been published at all or not in a usable form, corresponding researchers will be contacted to provide information.

Given that we have two objectives, two sets of search strings will be used in each database. According to table 1, the first set of keywords used to investigate cognitive processes and measures during walking with prosthesis (ie, objectives 1a and 1b) will be composed of four mandatory parts (\#1 AND \#2 NOT \#4)) and the second set of keywords used to investigate cognitive processes and measures during walking without prosthesis (ie, objectives $2 \mathrm{a}$ and $2 \mathrm{~b}$ ) will be composed of 3 mandatory parts (\#1 AND \#3 AND \#5)). Asterisks will be used to include alternative forms of the term (eg, attention* to include 
Part 1: (percept* OR attention* OR cogniti ${ }^{\star}$ OR workload OR "mental load" Limits the query to cognitive measures. OR "dual task" OR "dual-task")

Part 2: (((prosthe* OR amput*) AND ("lower-limb" OR "lower limb" OR "lower extremity" OR transfemoral OR "above knee" OR "above-knee")) OR "microprocessor knee" OR "prosthetic knee" OR "passive knee" OR "c-leg" OR "total knee" OR "power knee" OR "hydraulic knee")

Part 3: ("walk*" OR "gait")

Part 4: (cochlear OR "hearing loss" OR teeth OR dentures OR arthro* OR

"hip replacement" OR fracture OR exoskeleton)

Part 5: (assessment OR task OR measure* OR test)
Limits population to lower-limb amputee with prosthesis.

Limits study setting to walking.

Limits population to lower-limb amputee with prosthesis. Further limits the query to the cognitive measures. both attention and attentional) when this is allowed by the database. Quotation marks will be used for terms with multiple words (eg, "lower-limb"). The language will be limited to English. Additional searches in Chinese will also be conducted in search engines that contain nonEnglish information (eg, Google Scholar). One example in Chinese might be: “认知|注意|执行功能|知觉” AND “小 腿截肢|小腿假肢”. The actual format of the search strings will be adapted to for each database separately. The exact search strings and search details for each database will be recorded.

\section{Scoping exercise}

In order to demonstrate the effectiveness and sensitivity of our search strings, as recommended by CADIMA, ${ }^{16}$ we conducted a scoping exercise with a recent review paper in the relevant topic to check if the proposed search strings are suitable to retrieve the already known literature on relevant topics. The most comprehensive and relevant review (up to 2020) is the structured review on studies examining dual tasks by LL amputees, ${ }^{12}$ which covers 12 publications. We performed the scoping exercise on 19 February 2020. The set of 12 papers was used as a test library to check the new searches. We searched in WOS (including MEDLINE, BIOSIS, SciELO Citation Index), ProQuest Dissertations \& Theses Global, and EBSCO (including APA PsycINFO and CINAHL) with the following search string:

(percept* OR attention* OR cogniti* OR workload OR “mental load" OR "dual task" OR "dual-task") AND (( (prosthe* OR amput*) AND ("lower-limb" OR "lower limb" OR "lower extremity" OR transfemoral OR "above knee" OR “above-knee”)) OR "microprocessor knee” OR "prosthetic knee" OR "passive knee" OR "c-leg” OR "total knee" OR "power knee" OR "hydraulic knee”) NOT (cochlear OR "hearing loss" OR teeth OR dentures OR arthro* OR "hip replacement" OR fracture OR exoskeleton)

The obtained references were checked for the overlap with the test library. The results are listed in table 2. WOS delivered the best result. WOS found 11 of the 12 papers. Proquest and EBSCO found no papers. All databases combined returned 11 of 12 papers. The paper not obtained was:

- Hof AL, van Bockel RM, Schoppen T, et al. Control of lateral balance in walking. Experimental findings in normal subjects and above-knee amputees. Gait Posture 2007; 25(2): 250-258 (This is published online through PubMed/ScienceDirect but not indexed in the searched database).

The scoping exercise demonstrated that the proposed search string is suitable to return almost all of the relevant literature in the previous structured review. Articles that are not found with literature searches can be found by checking the references of previous reviews.

\section{Study inclusion criteria}

For the study screening for objectives $1 \mathrm{a}$ and $1 \mathrm{~b}$, articles will be included if fulfilling the following criteria of population, target setting and test. Population: a study should involve adult human participants with LL prosthesis, especially with above-knee prosthesis. Target setting: the study should involve a walking setting. Standing and balance setting will be excluded. Test: tasks or measures of attention, perception and cognition related to walking experiences will be included, including physiological measures. We will exclude cognitive assessment unrelated to gait or walking tasks, such as cognitive impairment screening tests and report on pain experience not during walking.

For the study screening for objectives $2 \mathrm{a}$ and $2 \mathrm{~b}$, articles will be included if fulfilling the following criteria of target setting and test. Target setting: the study should involve adult human participants in a physical walking test setting.

Table 2 Number of hits and references found with different literature databases as of 26 February 2020

\begin{tabular}{lllll}
\hline Database & WOS & Proquest & EBSCO & All combined \\
\hline Total hits & 810 & 90 & 266 & 1153 \\
$\begin{array}{l}\text { References } \\
\text { found (12 in }\end{array}$ & 11 & 0 & 0 & 11 \\
total) & & & & \\
\hline
\end{tabular}

WOS, Web of Science. 
Standing and balance setting will be excluded. Test: tasks or measures on attention, perception and cognition related to physical walking experiences will be included, including physiological measures. We will exclude cognitive assessment unrelated to gait or walking tasks, such as cognitive impairment screening tests and report on pain experience not during walking.

\section{Study screening mode}

\section{Screening articles: applying inclusion criteria}

The references retrieved by the literature searches will be imported to Refworks. Separate RIS file will be generated for each search engine to ensure transparency and repeatability of the search. Then, all the RIS files will be imported to CADIMA and combined into one database. Duplicates will be eliminated automatically and rechecked manually. In the first step, the inclusion criteria will be applied on titles and abstracts to remove unqualified references. The policy at this stage is to be as inclusive as possible. In the second step, one database with screened references from first step will be created in CADIMA. Full texts will be uploaded into CADIMA and then further filtered according to the inclusion criteria. At this stage, the reason for study exclusions will be documented.

\section{Screening articles: quality assurance process}

At the beginning of the screening process, a subset of studies (10\%, maximum 200 references) randomly drawn from the literature pool will be reviewed independently and parallelly by two team members with the predetermined inclusion criteria. The result of this subset will be analysed using Kappa statistics by CADIMA (http://www. vassarstats.net/kappa.html). Studies received different exclusion opinions between reviewers will be documented and discussed in the review team. If the kappa value is below $0.6^{17}$, an agreed strategy will be developed, and inclusion criteria will be refined and tested to improve reviewer agreement and to minimise discrepancies in the screening process.

\section{Critical appraisal of included studies}

For each study fulfilling the inclusion criteria of the scoping review after full-text screening, the reliability will be evaluated by judging the risk to systematic bias

Table 3 Draft data extraction sheet

1. Bibliographical information

2. Method_Sample
Article_id

Author

Publication_year

Title

Type of publication (peer-reviewed journal, non-peer-reviewed journal, book, grey literature)

Data location

Sample size

Population (with or without prosthesis)

Sampling method

Age

Experimental design

Randomised or matched allocation

Counterbalance/randomisation

Baseline included or not

Practice session included or not

\begin{tabular}{ll} 
4a. Method_Cognitive measures_quantatitive & Cognitive measure description \\
& Type of cognitive function \\
& Type of measures \\
& Type of walking setting \\
& Task prioritisation \\
& Task modality \\
& Cognitive quantification \\
& Cognitive measure output \\
& Any emergent theme/information during extraction that is relevant to the \\
4b. Method_Cognitive measures_qualitative & successful quantification of cognition during walking will be noted. \\
\hline 5. Other & Study funding \\
\hline
\end{tabular}


Table 4 Timeline for protocol and scoping review

\begin{tabular}{|c|c|c|c|c|c|c|c|}
\hline Month & $\begin{array}{l}\text { Feb-Mar } \\
2020\end{array}$ & $\begin{array}{l}\text { Apr-May } \\
2020\end{array}$ & $\begin{array}{l}\text { Jun-Sep } \\
2020\end{array}$ & $\begin{array}{l}\text { Oct 2020- } \\
\text { Jan } 2021\end{array}$ & $\begin{array}{l}\text { Feb-Mar } \\
2021\end{array}$ & $\begin{array}{l}\text { Apr } \\
2021\end{array}$ & Author involved \\
\hline Writing protocol & & & & & & & All authors \\
\hline Screening & & & & & & & JY, EC, JF \\
\hline Data extraction & & & & & & & JY, EC, JF \\
\hline
\end{tabular}

as low, high or uncertain. The systematic biases that are usually considered are selection bias, performance bias, detection bias, attrition bias and reporting bias. ${ }^{17}$ Given the purpose of scoping review is slightly different from systematic reviews in that we are focusing on the measures rather than the outcomes of the study, biases in selection, performance and attrition are not relevant in this review. As a result, we only will consider reporting bias (eg, preferential reporting of positive outcomes or method details). Study funding information will be extracted during data extraction and evaluated by two reviewers. Studies will be considered as higher risk when conducted by institutions/organisations/companies with a commercial, political or ideological interest in a certain outcome of their study (eg, prosthesis company might not publish cognitive results showing adverse effects of their own product).

\section{Data extraction strategy}

The variables that will be extracted are listed in table 3 . The data will be recorded in CADIMA. Information on how data will be extracted or obtained will also be given. If the presented measures are not detailed enough, authors will be contacted to provide the details. The following rules will be followed during data extraction:

- Baseline measures should be a similar or the same measure as implemented in the experimental or training phase.

- Only when the participant was explicitly asked to prioritise (eg, 'prioritise', 'focus on' and so on) the cognitive task or walking task, the task is counted as prioritised.

- If according to the measure description it is possible for the cognitive measure to generate computable output to directly indicate a person's cognitive performance during walking, it is counted as quantifiable. For example, if a person is asked to talk during walking but the cognitive load added from talking was not manipulated or cannot be quantifiable, this task is counted as unquantifiable.

- Cognitive measure output is counted as existing when the data are analysed and reported in the results section. For example, if a dual task is used but only gait performance is measured and reported, then it is counted as not having output.
A random selection (25\%) of the extracted data will be checked by a second member of the review team.

\section{Patient and public involvement}

Patients or the public will not be directly involved in the design, or conduct, or reporting, or dissemination plans of our research.

\section{DATA ANALYSIS}

All identified studies will be grouped by the codes in each variable. The results of the grouped variables will be presented in forms of text, figures and tables. Specifically, the major outcome for objectives $1 \mathrm{a}$ and $2 \mathrm{a}$ is the type of cognitive function and the results will describe (1) what cognitive functions have been assessed during walking with prosthesis and what is the percentage and frequency; (2) which additional cognitive functions have been assessed during walking without using prosthesis and which are the commonly assessed ones. The major quantitative outcomes for objectives $1 \mathrm{~b}$ and $2 \mathrm{~b}$ are the cognitive measures' characteristics (eg, types of cognitive measure, modality of the task, whether quantifiable or not), which will be discussed together with other secondary outcomes such as study year, sample characteristics, walking setting type, experimental design characteristics (eg, task prioritisation, whether baseline is measured, whether there is practice sessions) to answer questions such as whether certain measures are limited to certain walking setting, whether it has been assessed in various samples, and experimental design considerations related to using certain measures. A qualitative synthesis of the advantages and disadvantages of existing cognitive measures during walking will be analysed based on the detailed cognitive measure description together with other methodological-related variables and organised in identified themes. A narrative discussion of the current application status and gaps of cognitive performance assessment from both quantitative and qualitative synthesis and suggestions on cognitive measure selection, methodological considerations and future directions for empirical efforts will be provided.

\section{TIMELINE}

Table 4 shows the timeline for the whole scoping review process. 


\section{ETHICS AND DISSEMINATION}

This scoping review will evaluate the measures used in previous studies that have been conducted and does not require ethical approval. The results will contribute to the advancement of prosthesis tuning processes by reviewing the application status of current cognitive measures during walking with and without prosthesis and laying the foundation for developing new cognitive measures during walking. The results will be disseminated through outlets such as conferences and journals.

Contributors JF is the guarantor. All authors have contributed to the study design. JY led the preparation and writing of the protocol, jointly conceived the idea for the project and contributed to the development of research questions. EC contributed to the writing of the Method section and provided feedback on protocol drafts. ML contributed to the writing of the Introduction section and provided feedback on protocol drafts. HH jointly led the supervision of the project, the writing of the Introduction section and provided feedback on protocol drafts. JF led the supervision of the manuscript preparation, jointly conceived the idea for the project, contributed to the development of research questions and provided feedback on protocol drafts. All authors read and approved the final manuscript.

Funding This work was supported by the US National Science Foundation (NSF), M3X-Mind, Machine, Motor program, grant number 1926998.

Competing interests None declared.

Patient and public involvement Patients and/or the public were not involved in the design, or conduct, or reporting, or dissemination plans of this research.

Patient consent for publication Not required.

Provenance and peer review Not commissioned; externally peer reviewed.

Open access This is an open access article distributed in accordance with the Creative Commons Attribution Non Commercial (CC BY-NC 4.0) license, which permits others to distribute, remix, adapt, build upon this work non-commercially, and license their derivative works on different terms, provided the original work is properly cited, appropriate credit is given, any changes made indicated, and the use is non-commercial. See: http://creativecommons.org/licenses/by-nc/4.0/.

ORCID iD

Jing Yuan http://orcid.org/0000-0001-9178-3896

\section{REFERENCES}

1 Ziegler-Graham K, MacKenzie EJ, Ephraim PL, et al. Estimating the prevalence of limb loss in the United States: 2005 to 2050. Arch Phys Med Rehabil 2008;89:422-9.
2 Windrich M, Grimmer M, Christ O, et al. Active lower limb prosthetics: a systematic review of design issues and solutions. Biomed Eng Online 2016;15:140.

3 Tucker MR, Olivier J, Pagel A, et al. Control strategies for active lower extremity prosthetics and orthotics: a review. J Neuroeng Rehabil 2015;12:1.

4 Brandt A, Wen Y, Liu M, et al. Interactions between Transfemoral amputees and a powered knee prosthesis during load carriage. Sci Rep 2017;7.

5 Huang $\mathrm{H}$, Crouch DL, Liu M, et al. A cyber expert system for autotuning powered prosthesis impedance control parameters. Ann Biomed Eng 2016;44:1613-24.

6 Wen Y, Si J, Brandt A, et al. Online reinforcement learning control for the personalization of a robotic knee prosthesis. IEEE Trans Cybern 2020;50:1-11.

$7 \mathrm{Kim} \mathrm{M}$, Ding Y, Malcolm P, et al. Human-in-the-loop Bayesian optimization of wearable device parameters. PLoS One 2017;12:9,e0184054.

8 Caputo JM, Adamczyk PG, Collins SH. Optimizing prosthesis design to maximize user satisfaction using a tethered robotic ankle-foot prosthesis. Proceedings of Dynamic Walking, 2015:21-4.

9 Pirouzi G, Abu Osman NA, Eshraghi A, et al. Review of the socket design and interface pressure measurement for transtibial prosthesis. ScientificWorldJournal 2014;2014:1-9.

10 Dillingham TR, Pezzin LE, MacKenzie EJ, et al. Use and satisfaction with prosthetic devices among persons with trauma-related amputations: a long-term outcome study. Am J Phys Med Rehabil 2001;80:563-71.

11 Horgan O, MacLachlan M. Psychosocial adjustment to lower-limb amputation: a review. Disabil Rehabil 2004;26:837-50

12 Morgan SJ, Hafner BJ, Kartin D, et al. Dual-Task standing and walking in people with lower limb amputation: a structured review. Prosthet Orthot Int 2018;42:652-66.

13 Hasanzadeh S, Esmaeili B, Dodd MD. Examining the relationship between construction workers' visual attention and situation awareness under fall and tripping hazard conditions: using mobile eye tracking. J Constr Eng Manag 2018;144:04018060.

14 Brantley JA, Luu TP, Nakagome S. Towards the development of a hybrid neural-machine interface for volitional control of a powered lower limb prosthesis [abstract]. International Symposium on Wearable Robotics and Rehabilitation; 2017 Nov. 5-8, Houston TX, USA, 2017:1.

15 Knaepen K, Marusic U, Crea S, et al. Psychophysiological response to cognitive workload during symmetrical, asymmetrical and dualtask walking. Hum Mov Sci 2015;40:248-63.

16 Kohl C, Kohl C, McIntosh EJ, et al. Online tools supporting the conduct and reporting of systematic reviews and systematic maps: a case study on CADIMA and review of existing tools. Environ Evid 2018;7:1-17.

17 Meissle M, Naranjo SE, Kohl C, et al. Does the growing of Bt maize change abundance or ecological function of non-target animals compared to the growing of non-GM maize? A systematic review protocol. Environ Evid 2014;3:7. 